\title{
Use of Multilayer Correction Procedures to Improve the Quantitative Electron Microprobe Analyses of Coated Insulating Specimens
}

\author{
J.T. Armstrong* \\ * Geophysical Laboratory, Carnegie Institute of Washington, 5251 Broad Branch Road, N.W., \\ Washington, DC 20015-1305
}

Evaporating or sputtering thin layers of conducting materials onto specimens for electron microscopy and microbeam analysis have long been employed to improve electrical and thermal conductivity, signal to noise ratio, and spatial resolution [1]. However, such surface coatings affect the relative emitted $\mathrm{x}$-ray intensities and complicate quantitative analyses. Traditionally, when performing quantitative microprobe analysis, surface coatings are limited to thin layers of light elements (usually carbon), and samples and standards are coated at the same time or with thickness monitors to insure that both have the same overlayer thickness [2]. When such procedures are not followed, the errors in quantitative analysis can easily exceed 5\%, and even when carefully undertaken, multi-percent level variations in analytical results can still be caused by spatial variations in coating thickness $[3,4]$.

Many practical applications of electron microprobe analysis do not involve the samples and standards having the same coating thicknesses, or sometimes even having samples and standards coated with the same material. Examples include: already coated samples brought to a lab for analysis; samples that cannot be coated being analyzed using coated standards; samples to be analyzed for carbon using C-coated standards; samples coated with elements like $\mathrm{Cr}$, $\mathrm{Pt}$ and $\mathrm{Au}$ for high resolution imaging, SIMS analysis, etc., being analyzed using C-coated standards. Errors produced in such cases often exceed 10\%. Advances in recent years in the electron microprobe analysis of multi-layer samples using multiple accelerating potential EPMA with algorithmic or Monte Carlo correction procedures lend hope that routine, accurate quantitative methods can be utilized to correct for coating composition and thickness in these cases as well [5].

These methods have proven very successful in accurately determining the thicknesses and compositions of surface layer(s) on substrates that are usually of known composition, but there is less evidence that they provide a robust means of accurately determining the composition of an unknown substrate underneath one or more layers of known composition and approximate thicknesses. In the former case, correction involves determining the relative intensity emitted by an isolated thin film of given thickness with respect to a bulk material of the same composition and then correcting for additional intensity due to electron backscattering and x-ray fluorescence from the substrate - factors that have been measured experimentally and successfully simulated by Monte Carlo methods. In the latter case, correction involves determination of the range of energy losses and changes of direction that electrons undergo as they pass through the surface layer(s), their effects on x-ray production relative to electron bombardment with a single energy and orientation, then correction for x-ray absorption and fluorescence by the overlayer(s). These factors are difficult to measure directly and more dependent on the scattering model used in Monte Carlo simulations.

We have used multilayer corrections procedures to back-correct analytical data in our laboratory involving samples and standards with greatly differing carbon-coat thicknesses and heavy-metal 
coated samples analyzed relative to carbon coated standards. We have utilized both the algorithmic corrections contained in the GMRFilm [6] and CITZAF [7,8] programs and the Monte Carlo simulation procedures contained in the CASINO program [9].

Results for samples and standards having significantly different thickness of carbon coats are very promising - even for elements having low overvoltage or heavily absorbed by the carbon coat. For example. an already $\mathrm{C}$-coated iron oxide sample was brought in for oxygen and iron analysis to determine the oxide stoichiometry (expected to be wustite, $\mathrm{Fe}_{1-\mathrm{x}} \mathrm{O}$ ). The samples had much thicker carbon coats than the standards (ranging from 30 to $50 \mathrm{~nm}$ compared to $15 \mathrm{~nm}$ for the standards). The analyses without correction for the coat thickness variation summed between $92-95 \%$ and the stoichiometry varied with $\mathrm{Fe}>\mathrm{O}$. Analyzing for $\mathrm{C}$ and correcting for its thicknesses resulted in the analyses summing between 99.5-100.5 with Fe:O ratios varying from 0.98:1 to 1:1. Both Monte Carlo and algorithmic corrections gave very similar results with insignificant differences among the various algorithmic models employed.

Analyses of high-Z-coated samples relative to carbon coated standards give more variable results with disagreement among the various models employed. In a typical example, a Pt-coated glass (Na 3.53 wt. \%, Mg 2.85\%, Al 0.69\%, Si 37.12\%, K 0.84\%, Ca 5.00\%, O 49.98\%) was analyzed at 10 and $15 \mathrm{keV}$ relative to a series of C-coated samples. The standards had a carbon coat thickness of $\sim 16.0 \mathrm{~nm}$. The carbon coat on the glass sample was determined to be $\sim 11 \mathrm{~nm}$ thick. The Pt-coated sample had a thickness of $\sim 10 \mathrm{~nm}$. All of the layer-thickness-processed analyses of the C-coated sample summed to between 99 and $100 \%$ with less than $2 \%$ relative variation from the nominal composition for any element. The conventional ZAF analysis of the Pt-coated sample yielded results totaling $\sim 79 \%$, with $35 \% \mathrm{O}, 32.5 \% \mathrm{Si}$, and $4 \% \mathrm{Ca}$. Analyses using the Casino Monte Carlo processing summed be 100 to $101.5 \%$, with higher than nominal $\mathrm{O}$ and $\mathrm{Na}$ concentrations $(\sim 50.6$ and 3.9\%). The various GMRFilm algorithms all produced analyses that summed low ( $96 \%$ for one of the more commonly used corrections and $91 \%$ for another). They were several $\%$ low for each of the elements, with relative concentrations similar to the nominal values. The results suggest that more testing and correction refinement need to be employed for analysis of heavy-metal-coated samples using C-coated specimens; but the Monte Carlo results, at least, are promising that a robust method can be found. Computer software on commercial electron microbeam instruments need to be updated to enable routine use and continued development of multi-layer analysis.

\section{References}

[1] J.I. Goldstein et al., Scanning Electron Microscopy and X-ray Microanalysis, $3^{\text {rd }}$ Ed., Kluwer/Plenum, New York, 2003.

[2] D.M. Kerrick et al., Amer. Mineral. 58 (1973) 920.

[3] J.T. Armstrong, Microbeam Anal. 2 (Suppl. 1) (1993) 13.

[4] M. Raudsepp, Canad. Mineral. 33 (1995) 203.

[5] J.A. Small et al., in Handbook of X-ray Spectrometry, $2^{\text {nd }}$ ed., R. Van Grieken et al., eds., Marcel Dekker, New York (2002) 811.

[6] R.A. Waldo, in Microbeam Anal. 2001, D.G. Howitt, Ed., (2001) 45.

[7] J.T. Armstrong, Microbeam Anal. 4 (1995) 177.

[8] J.T. Armstrong, in Electron Probe Quantitation, K.F.J. Heinrich et al., eds., Plenum, New York (1991) 261.

[9] D. Drouin et al., Scanning 29 (2007) 92. 